Jahrbuch der Raabe-Gesellschaft 2018 



\section{Jahrbuch der Raabe-Gesellschaft 2018}

Im Auftrag des Vorstands

Herausgegeben von

Andreas Blödorn und Madleen Podewski

\section{DE GRUYTER}


Anschriften der Herausgeber:

Professor Dr. Andreas Blödorn, Westfälische Wilhelms-Universität Münster, FB Philologie, Germanistisches Institut (Neuere deutsche Literatur und Medien), Schlossplatz 34, 48143 Münster

E-Mail: andreas.bloedorn@uni-muenster.de

(= Redaktionsanschrift für Aufsätze)

PD Dr. Madleen Podewski, Freie Universität Berlin, FB Philosophie und Geisteswissenschaften, Institut für Deutsche und Niederländische Philologie, Habelschwerdter Allee 45, 14195 Berlin E-Mail: madleen.podewski@fu-berlin.de (= Redaktionsadresse für Literaturberichte)

Mit freundlicher Unterstützung durch

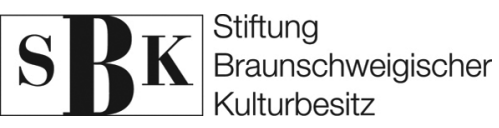

\section{Stadt Braunschweig \\ ang Dezernat für Kultur und Wissenschaft}

ISSN 0075-2371

e-ISSN 1865-8857

\section{Library of Congress Cataloging-in-Publication Data}

A CIP catalog record for this book has been applied for at the Library of Congress.

\section{Bibliografische Information der Deutschen Nationalbibliothek}

Die Deutsche Nationalbibliothek verzeichnet diese Publikation in der Deutschen Nationalbibliografie; detaillierte bibliografische Daten sind im Internet über http://dnb.dnb.de abrufbar.

(C) 2019 Walter de Gruyter GmbH, Berlin/Boston

Satz: jürgen ullrich typosatz, Nördlingen

Druck und Bindung: CPI Books GmbH, Leck

@ Gedruckt auf säurefreiem Papier

www.degruyter.com 


\section{Inhalt}

Vorbemerkung und Dank — 1

\section{Aufsätze}

Ulrike Vedder

Interieurs und Exterieurs. Räume und ihre Wirklichkeit in Wilhelm Raabes

Literatur -5

Cornelia Blasberg

Vor dem Realismus? Themen und Schreibverfahren in Annette

von Droste-Hülshoffs literarischen Texten - 23

Stephan Brössel

Die Phase zwischen Goethezeit und Realismus als Problemfall der Literaturgeschichte. Am Beispiel der Novellistik Theodor Mügges — 42

Stefan Tetzlaff

Überlegungen zu ,prekären' Literaturen am Beispiel von Theodor Fontane, Ernst Eckstein und Paul Lindau $\longrightarrow 70$

Ingrid Steiger-Schumann

Leopold Komperts Vision von ,Nachbarschaft'. Jüdisch-christliche

Liebesbeziehungen im Werk des böhmischen Schriftstellers — 91

Corinna Sauter

PROVERSA - „Oder umgekehrt“. Wilhelm Raabes (Literatur-)Satire „Deutscher Mondschein“ (1872/1873) als Programmschrift der Prosa — 109

\section{Literaturberichte und Bibliographie}

Rainer Barbey

Das Wissen der Poesie. Lyrik, Versepik und die Wissenschaften im 19. Jahrhundert. Hg. von Henning Hufnagel und Olav Krämer. Berlin [u. a.] $2015-197$ 
Hans-Joachim Hahn

Klaus Deterding: „Unter Thränen wachse ich immer mehr aus meinem Antisemitismus heraus“. Seitenhiebe auf Juden und das ,jüdische“ in der europäischen Literatur des 19. Jahrhunderts. Berlin $2016-203$

Dorit Müller

Archiv/Fiktionen. Verfahren des Archivierens in Literatur und Kultur des langen 19. Jahrhunderts. Hg. von Daniela Gretz und Nicolas Pethes. Freiburg/Breisgau, Berlin, Wien $2016-209$

Julia Menzel

Vergessene Konstellationen literarischer Öffentlichkeit zwischen 1840 und 1885. Hg. von Katja Mellmann und Jesko Reiling. Berlin/Boston $2016-218$

Madleen Podewski

Dynamik und Dialektik von Hoch- und Trivialliteratur im deutschsprachigen Raum im 18. und 19. Jahrhundert. I. Die Dramenproduktion. II. Die Erzählproduktion. Würzburg $2015-223$

Lisa Behrendt

Raabe-Bibliographie $2018-231$

Anschriften der Beiträgerinnen und Beiträger — 235 